\title{
INSTRUCTIONAL DESIGN OF KNOWLEDGE NUGGETS
}

\author{
Christian Ploder, Lisa Ehrhardt, Laura Gamper, \& Christoph Hazy \\ Management Communication \& IT, MCI, Innsbruck (Austria)
}

\begin{abstract}
Throughout their careers, all students experience the same set of learning routines within the same time frame and following the same pedagogical approach based on identical materials. In contrast, Knowledge Nuggets provide students with different opportunities to achieve specific learning outcomes through self-directed learning activities. An individual student's skill inventory could improve the full individualization of the Knowledge Nugget delivery at the start of each class. Knowledge Nuggets are considered learning materials organized within small, defined topics. This granularity makes the content easier to consume at an individual pace. Depending on their scope and size, these Knowledge Nuggets can vary. The level does not indicate the amount of content or the shared knowledge's difficulty but how the learning is prepared and conveyed.

The simplest way (level 1) to share knowledge is only to provide text within a document. The next higher level 2 includes a slideshow explained in more detail by playing an audio podcast - so the combination of visual with audio should attend two senses in parallel. The slides contain images or diagrams in addition to keywords. Video tutorials are the most advanced ways to share knowledge. This type of tutoring allows instructors to visually illustrate content to minimize complexity by combining text, speech, images, and animation. With level 3 (video tutorials), it is possible to address more senses simultaneously.

However, all three levels offer the same characteristics: practicality, reproducibility, and manageable time. These characteristics are of great relevance to be accepted as training methods in any educational program. The progressive redistribution of dimensions occurs in elaboration, the use of technology, and the cognitive load. The paper aims to determine why the different levels are essential for the overall picture and how the measurement of learning success can be measured.
\end{abstract}

Keywords: Knowledge nuggets, knowledge sharing, cognitive load.

\section{Introduction}

Digitalization is proceeding in the economy and society, and the educational sector is experiencing far-reaching changes. Higher educational institutions are increasingly opening up towards the potential of digitization. With it comes the expectation that research and administration, and instruction will improve quality and become more professional. Although the overall concept and degree of implementation can vary from institution to institution, digitization also requires structural preconditions and technological implementations and comes with its challenges. (Gilch et al., 2019)

With the Corona pandemic causing massive restrictions on public life since spring 2020 and in-person lectures being banned, digital instruction is coming to the fore. To continue sustaining education, the short-term development and use of digital teaching formats are essential.

One approach to share knowledge is by providing Knowledge Nuggets (Ploder et al., 2020), created for self-directed learning. Knowledge Nuggets are digitally prepared learning materials that are organized within small thematic categories. (Knowledge Nuggets are deeply related to E-Learning.) The learning content can be provided in different ways. For example, as a video, a slideshow, a web page, or a podcast. (Bailey et al., 2006; Shaw et al., 2014)

These Knowledge Nuggets differ based on an individual's cognitive ability to elaborate/ process them and, therefore, be classified into different levels. The term cognitive ability also includes the concept of cognitive load. A higher stress level indicates a more complicated and unpleasant perception of information intake (Sweller, 1994). This paper focuses on elaborating the factors to define these levels, separate them from each other and thus have to be considered for planning differentiated Knowledge Nuggets. The initial idea was to start with three different levels respectively Knowledge Nuggets based on their relevance and frequency of use in higher education. In section 3 , the explanation and all the factors are described.

After the first section with a brief introduction, section 2 outlines the authors' literature review and framing and the primary resources. The research focused in general on the thematic area of designing digital learning content. The literature sources were used to identify the relevant factors for developing knowledge nuggets and differentiating the different levels by design. The determining factors were then 
specified in more detail in the Instructional Design and integrated into an overall framework in section 3. Section 4 continues by describing opportunities for testing the three design levels to determine which level offers the highest potential. In the end, a review of limitations is given in section 5.

\section{Theoretical background}

This section represents a precise analysis of the current state of research and selects suitable approaches to identify studies and other sources comparing instructional design principles for Knowledge Nuggets. The authors elaborated a systematic literature review. As a guideline, the NHS Centre for Reviews and Dissemination (2009) approach was adapted to this work's requirements. The selected phases are outlined in table 1.

Table 1. Search terms used in the systematic literature review.

\begin{tabular}{|c|l|l|}
\hline Phase No. & Phase & Steps \\
\hline 1 & Planning the review & $\begin{array}{l}\text { Purpose of the literature review } \\
\text { Developing the review protocol }\end{array}$ \\
\hline 2 & Conducting the review & $\begin{array}{l}\text { Searching the literature and assessing studies for relevance } \\
\text { Assessing and grading studies for validity (quality) } \\
\text { Extracting data from the selected studies } \\
\text { Synthesizing data from the selected studies }\end{array}$ \\
& & Documentation and interpretation of the results \\
\hline 3 & Reporting &
\end{tabular}

Phase 1 starts with the definition of the framework for the analysis and is followed by the formalization of the review protocol, which determines the methods used to perform the review. To formulate a search strategy and create this framework, gaining familiarity with a deep understanding of the study field through relevant contributions and discussions is needed. Through topic-related preliminary research, carried out through the Google Scholar database and a general internet search, it was possible to determine the first state of knowledge for the topic to be worked on. Fundamental studies found at this stage were directly incorporated into the collection. Ongoing the systematic literature review process, the Google Scholar database was used as a basis for identifying qualified sources. The further specialization by including relevant search terms refined after the initial search helped to focus the analysis's scope. The summarized list of all used search terms is represented in table 2.

Table 2. Search terms for the systematic literature review.

\begin{tabular}{|l|l|}
\hline Keywords \\
\hline Design of video; cognitive load; learning performance; multimedia learning & Signaling; multimedia learning \\
\hline Learning from text and video; instructional design; multimedia learning & Emotional design; multimedia learning \\
\hline
\end{tabular}

Besides these mentioned criteria, a further characteristic was established regarding the publication period and the full-text availability. To focus on the most recent research on this topic, only full-text accessible articles from 2014 are included. The items were sorted in the relevance ranking of Google Scholar. A limit of the first 50 references was set to be reviewed if the search results in an excessively high number of hits.

Based on this entire collection of different criteria and the research protocol development, proceed to phase 2, illustrated in figure 1. Generally, in phase 2, searching the literature is executed. More precisely, it identifies potential studies and assesses the quality of these ongoing in the procedure. If the database search matches the outlined requirements and the search terms, validation is initially required by removing all presented duplicates. All references that had the identical title and author and published within the same year were excluded-moving on to the stage "First level screening", by reading just the title and abstract of the candidate studies and then deciding whether to include or exclude the study. In this way, studies that are not related to the topic can be removed. Whether to include an article, the full document was retrieved (Second level screening). The result represented as a pool of 16 studies is ready for the data extraction and quality assessment and can be synthesized to finalized.

Figure 1. Study selection.

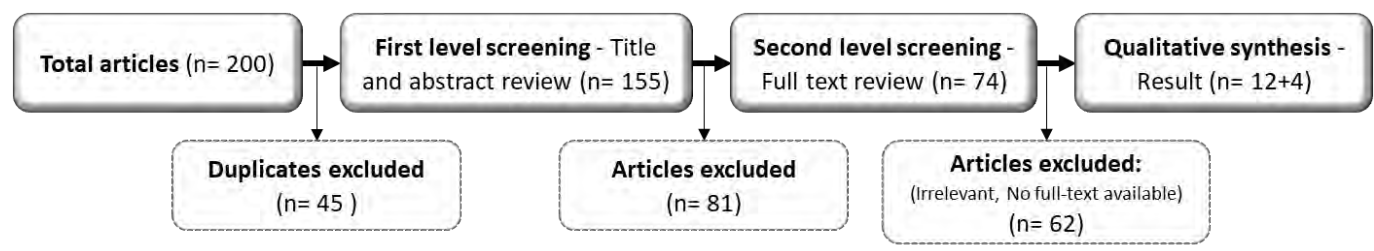


In the final stage in phase 3, the last documentation and the interpretation of the findings is carried out. Table 3 shows illustrate all selected relevant literature with title, Authors, and names.

Table 3. Overview Literature.

\begin{tabular}{|c|c|c|c|}
\hline No & Title & DOI & Year \\
\hline 1 & $\begin{array}{l}\text { A meta-analysis of how signaling affects learning with } \\
\text { media }\end{array}$ & https://doi.org/10.1016/j.edurev.2017.11.001 & 2018 \\
\hline 2 & $\begin{array}{l}\text { Assessing the effects of different multimedia materials on } \\
\text { emotions and learning performance for visual and verbal } \\
\text { style learners }\end{array}$ & https://doi.org/10.1016/j.compedu.2012.05.006 & 2012 \\
\hline 3 & Benefits of emotional design in multimedia instruction & https://doi.org/10.1016/j.learninstruc.2014.02.004 & 2014 \\
\hline 4 & $\begin{array}{l}\text { Cognitive load in multimedia learning environments: A } \\
\text { systematic review }\end{array}$ & https://doi.org/10.1016/j.compedu.2019.103618 & 2019 \\
\hline 5 & $\begin{array}{l}\text { Cognitive load theory and multimedia learning, task } \\
\text { characteristics and learning engagement: The Current State } \\
\text { of the Art }\end{array}$ & https://doi.org/10.1016/j.chb.2010.05.003 & 2011 \\
\hline 6 & $\begin{array}{l}\text { Effective Educational Videos: Principles and Guidelines } \\
\text { for Maximizing Student Learning from Video Content }\end{array}$ & https://doi.org/10.1187/cbe.16-03-0125 & 2016 \\
\hline 7 & $\begin{array}{l}\text { Effects of different video lecture types on sustained } \\
\text { attention, emotion, cognitive load, and learning } \\
\text { performance }\end{array}$ & https://doi.org/10.1016/j.compedu.2014.08.015 & 2015 \\
\hline 8 & $\begin{array}{l}\text { Emotional design in multimedia learning: Differentiation } \\
\text { on relevant design features and their effects on emotions } \\
\text { and learning }\end{array}$ & https://doi.org/10.1016/j.chb.2014.11.009 & 2015 \\
\hline 9 & $\begin{array}{l}\text { Emotional design in multimedia learning: Effects of shape } \\
\text { and color on affect and learning }\end{array}$ & https://doi.org/10.1016/j.learninstruc.2013.02.006 & 2014 \\
\hline 10 & How multimedia can improve learning and instruction & https://doi.org/10.1017/9781108235631.019 & 2019 \\
\hline 11 & $\begin{array}{l}\text { Instructional animation versus static pictures: A meta- } \\
\text { analysis }\end{array}$ & https://doi.org/10.1016/j.learninstruc.2007.09.013 & 2007 \\
\hline 12 & $\begin{array}{l}\text { Learning from Examples: Instructional Principles from the } \\
\text { Worked Examples Research }\end{array}$ & https://doi.org/10.3102/00346543070002181 & 2000 \\
\hline 13 & $\begin{array}{l}\text { Learning from the text, video, or subtitles: A comparative } \\
\text { analysis }\end{array}$ & https://doi.org/10.1016/j.compedu.2020.104034 & 2021 \\
\hline 14 & $\begin{array}{l}\text { Signaling text-picture relations in multimedia learning: A } \\
\text { comprehensive meta-analysis }\end{array}$ & https://doi.org/10.1016/j.edurev.2015.12.003 & 2016 \\
\hline 15 & Thirty years of research on online learning & https://doi.org/10.1002/acp.3482 & 2018 \\
\hline 16 & $\begin{array}{l}\text { Trends and issues in multimedia learning research in } \\
\text { 1996-2016: A bibliometric analysis }\end{array}$ & https://doi.org/10.1016/j.edurev.2019.100282 & 2019 \\
\hline
\end{tabular}

\section{Instructional design}

In this section, the collected findings from the systematic literature review provide a framework for planning differentiated Knowledge Nuggets by design. The three levels which correspond with the given literature can be named: (i) text within a document, (ii) slideshow with an added audio podcast, and (iii) video tutorials, the various principles and techniques were applied, and factors identified. The designed model depicted in table 4 provides an overview of the relevant characteristics to obtain the desired Knowledge Nugget at a certain level. All the factors and their expressions in explained in the following subsections. It is worth mentioning that all levels are assumed to provide the same quality and the same amount of knowledge.

Table 4. Relevant factors for the Instructional Design.

\begin{tabular}{|l|l|l|l|}
\hline Factors & Level 1 & Level 2 & Level 3 \\
\hline Visual Elements & & Static images & Dynamic images/animations \\
\hline Recipient engagement & Self-regulated & Self-regulated & Controlled \\
\hline Language Style & Formal Style & Conversational style & Conversational style \\
\hline Sensory Modalities & Visual sense & Auditory + visual senses & Auditory + visual senses \\
\hline
\end{tabular}

Visual Elements - One of the factors that differentiate the Knowledge Nuggets and, therefore, the levels from one another are visual elements. In general, visual features are considered to be, e.g., graphics, images, diagrams, and animations (Li et al., 2019) on top of text. Since visual elements do not support a pure continuous text, it strongly differs among the other levels. For instance, slideshows, compared to level 1, often contain a variety of static visual elements, such as diagrams and images. However, according to Lewalter (1997), static images can sometimes cause misinterpretations and result in an inaccurate understanding or prospect. Moreover, misinterpretations may increase a participant's cognitive load.

Besides static graphics, dynamic images like animations are familiar. According to Rieber and Kini (1991), an animation is formed by a series of fast-changing computer screen visuals that create the impression of motion to a viewer. These are primarily used for video productions, representing level 3. However, it should be noted that animations do not represent permanent information but a current capture 
(Hegarty, 2004). The risk of misinterpreting images can be decreased by (using) animations, as they facilitate procedural explanations that can lower an individual's cognitive load. To visualize the same complex process differently, multiple static graphics would be required. (Höffler \& Leutner, 2007)

Recipient engagement - A further factor that distinguishes each level is the active regulation of Knowledge Nuggets by the recipient. Examples include repeating or skipping information and regulating the speed of information intake. Regarding videos, the transmission of information is preferably controlled by the sender since the receiver does not influence the rate of animations or the speaker's performance speed. Repeating or skipping information in a controlled way is more difficult in this case since videos usually do not have well-defined section headings/ delineated sections. A slideshow including an integrated podcast, on the other hand, is more comfortable to regulate as the individual slides structure the presentation. The recipient proceeds with the presentation autonomously by browsing through the slides and can initiate the voice-over of each slide independently. In comparison to videos and slideshows, processing texts can be controlled by the recipients to a much greater extent. The reader has the opportunity to reread sentences or paragraphs, skip parts, and adjust his reading pace to his needs. Thus, the learning process is even more personalized. (Merkt et al., 2011; Tarchi et al., 2021)

Language Style - Moving on to the next factor, the authors examine the influence of language style. Principally, language is another crucial component of the transmitter-retriever model and represents the primary medium through which we can share knowledge. Changing narration from a formal textbook-like style to a more personalized conversational style in multimedia instruction has shown a significant effect on learning in the past; people may know more deeply (Mayer, 2002). Mayer describes this phenomenon as the personalization principle (Mayer, 2008). Mayer believes that using conversational language instead of formal language in multimedia teaching will encourage retrievers to establish social relationships with the narrator. That leads to greater participation and effort. For instance, while explaining, make instead use of "your" alternatively to "the" when involving the retriever or the use of "I" to point the narrator's viewpoint. While the text in level 1 is only a composite of words and therefore has to be presented more formally, level 2 and level 3 can use the personalization principle. Regardless of the version, the same factual information is always transmitted. (Mayer, 2018)

Sensory Modalities - Another factor of level differentiation is the form/ expression of visual and auditory stimuli/ allures. Based on how Knowledge Nuggets are set up, the cognitive load resp. The stimuli of the participants are strained differently. According to Mayer (2001), individuals possess separate channels to process auditory/verbal and visual/pictural information. The continuous text provided in the first level is processed in the vocal track. When consuming a slideshow, the load is divided into two channels. The spoken words and keywords on the slides are captured in the auditory/verbal channel. The static images are displayed in the visual/pictorial channel.

Regarding videos, the visual/pictorial channel is additionally claimed by animations. Referring to Mayer (2001), individuals only have limited capacity to process input material in these channels. Therefore, the channels should not be overloaded. While the cognitive load generated by texts arises from the verbal channel only, it is divided between both channels when viewing slideshows and videos. Consequently, the cognitive load is strained in different ways. That also influences the design of the considered Knowledge Nuggets. Thus, the only formatted continuous text is used in level 1. Since slideshows additionally use images and audio files for presentation, the text is limited to keywords on the slides. The unique characteristics of videos are the animations. These, in return, replace some verbal/auditory stimuli of slideshows. In conclusion, the Cognitive Load mustn't be overloaded in any of these levels. (Mayer, 2018).

To sum it up finally, this section provides an understanding of how the levels differ and indicates that this may significantly change the learning experience.

\section{Future research}

During the elaboration of the theoretical framework, further interesting considerations could lead to additional research soon. Creating differentiated Knowledge Nuggets should not only be based on the factors mentioned above but also on general design factors. Therefore, for an expanded approach, design aspects should be addressed and included. Moreover, the level factors identified in this paper only provide a theoretical policy regarding how levels differ from one another and how they influence the cognitive load of Knowledge Nugget recipients. To verify these factors and their effect in practical application, they can be tested by running an empirical study. Conducting such a study requires that differentiated Knowledge Nuggets are created based on the previously mentioned theoretical framework and can be provided digitally on suitable platforms, such as LMSs. Subjects may begin the learning process subsequently by retrieving the instructional materials under predetermined conditions. After performing the learning process, the stress level based on the Cognitive Load Theory can be measured using standardized scales. Questionnaires or tests are suitable for evaluating comprehension and reproducibility. The results given by these measurements may then indicate which types of levels, and thus which factors, should be utilized for learners such as students of higher education institutions to impart knowledge. 


\section{Limitations}

When interpreting the findings of this study, some limitations need to be taken into account. First, it has to be mentioned that the literature review was restricted to some specific journals within a certain period. Another factor is that the Knowledge Nuggets instructional design is not yet validated in a practical setting. Experts can be used in the validation process to minimize this impact in the future.

Furthermore, by designing the Knowledge Nuggets, the authors decided to prepare exactly three levels of Knowledge Nuggets. That is a limiting factor because it would be possible to split the nuggets into more detailed levels. The last limiting factor is that our Knowledge Nuggets are designed for digital learning. That means that the purpose of the nuggets is to work correctly on digital screens compared to the idea of differentiation of analog and digital systems based on (Singer \& Alexander, 2017).

\section{References}

Bailey, C., Zalfan, M. T., Davis, H. C., Fill, K., \& Conole, G. (2006). Panning for Gold: Designing Pedagogically-Inspired Learning Nuggets. Educational Technology \& Society, 9(1), 113-122. https://eric.ed.gov/?id=EJ836698

Gilch, H., Beise, A. S., Krempkow, R., M. Müller, Stratmann, F., \& Wannemacher, K. (2019). Digitalisierung der Hochschulen: Ergebnisse einer Schwerpunktstudie für die Expertenkommission Forschung und Innovation. Studien Zum Deutschen Innovationssystem Expertenkommission Forschung Und Innovation (EFI).

Hegarty, M. (2004). Dynamic visualizations and learning: getting to the difficult questions. Learning and Instruction, 14(3), 343-351. https://doi.org/10.1016/j.learninstruc.2004.06.007

Höffler, T. N., \& Leutner, D. (2007). Instructional animation versus static pictures: A meta-analysis. Learning and Instruction, 17(6), 722-738. https://doi.org/10.1016/j.learninstruc.2007.09.013

Lewalter, D. (1997). Lernen mit Bildern und Animationen: Studie zum Einfluss von Lernermerkmalen auf die Effektivität von Illustrationen. Pädagogische Psychologie und Entwicklungspsychologie. Waxmann.

Li, J., Antonenko, P. D., \& Wang, J. (2019). Trends and issues in multimedia learning research in 1996-2016: A bibliometric analysis. Educational Research Review, 28, 100282. https://doi.org/10.1016/j.edurev.2019.100282

Mayer, R. E. (2001). Multimedia learning. Cambridge University Press. https://doi.org/10.1017/CBO9781139164603

Mayer, R. E. (2002). Multimedia learning. Psychology of Learning and Motivation, 41, 85-139. https://doi.org/10.1016/S0079-7421(02)80005-6

Mayer, R. E. (2008). Applying the science of learning: Evidence-based principles for the design of multimedia instruction. The American Psychologist, 63(8), 760-769. https://doi.org/10.1037/0003066X.63.8.760

Mayer, R. E. (2018). Thirty years of research on online learning. Applied Cognitive Psychology, 33(2), 152-159. https://doi.org/10.1002/acp.3482

Merkt, M., Weigand, S., Heier, A., \& Schwan, S. (2011). Learning with videos vs. learning with print: The role of interactive features. Learning and Instruction, 21(6), 687-704. https://doi.org/10.1016/j.learninstruc.2011.03.004

NHS Centre for Reviews and Dissemination. (2009). CRD's Guidance for Carrying Out or Commissioning Reviews (2. ed.). Systematic reviews. York Publ. Services.

Ploder, C., Dilger, T., Bernsteiner, R. (2020) Knowledge-Nuggets based Self Learning - COVID-19 based Qualitative Research Lecture, ICERI2020 Proceedings, pp. 2389-2393. ISBN: 978-84-0924232-0

Rieber, L. P., \& Kini, A. (1991). Theoretical foundations of instructional applications of computer-generated animated visuals. Journal of Computer-Based Instruction, 18(3), 83-88.

Shaw, C., Larson, R., \& Sibdari, S. (2014). An Asynchronous, Personalized Learning Platform-Guided Learning Pathways (GLP). Creative Education, 05(13), 1189-1204. https://doi.org/10.4236/ce.2014.513135

Singer, L. M., \& Alexander, P. A. (2017). Reading on Paper and Digitally: What the Past Decades of Empirical Research Reveal. Review of Educational Research, 87(6), 1007-1041. https://doi.org/10.3102/0034654317722961

Sweller, J. (1994). Cognitive load theory, learning difficulty, and instructional design. Learning and instruction, 4(4), 295-312.

Tarchi, C., Zaccoletti, S., \& Mason, L. (2021). Learning from text, video, or subtitles: A comparative analysis. Computers \& Education, 160, 104034. https://doi.org/10.1016/j.compedu.2020.104034 